\title{
REVIEW \\ Pediatric solid tumor genomics and developmental pliancy
}

\author{
X Chen ${ }^{1}$, A Pappo ${ }^{2}$ and MA Dyer ${ }^{3,4}$
}

Pediatric solid tumors are remarkably diverse in their cellular origins, developmental timing and clinical features. Over the last 5 years, there have been significant advances in our understanding of the genetic lesions that contribute to the initiation and progression of pediatric solid tumors. To date, over 1000 pediatric solid tumors have been analyzed by Next-Generation Sequencing. These genomic data provide the foundation to launch new research efforts to address one of the fundamental questions in cancer biology - why are some cells more susceptible to malignant transformation by particular genetic lesions at discrete developmental stages than others? Because of their developmental, molecular, cellular and genetic diversity, pediatric solid tumors provide an ideal platform to begin to answer this question. In this review, we highlight the diversity of pediatric solid tumors and provide a new framework for studying the cellular and developmental origins of pediatric cancer. We also introduce a new unifying concept called cellular pliancy as a possible explanation for susceptibility to cancer and the developmental origins of pediatric solid tumors.

Oncogene (2015) 34, 5207-5215; doi:10.1038/onc.2014.474; published online 2 February 2015

\section{INTRODUCTION}

Childhood cancers are broadly classified on the basis of their anatomic location rather than common molecular, cellular or genetic features. Hematologic malignancies are cancers of the blood, bone marrow and the lymphoid system. Brain tumors are intracranial cancers of the central nervous system, and solid tumors are extracranial non-hematologic cancers. Pediatric solid tumors include carcinomas derived from epithelial cells (for example, adrenocortical carcinoma) and sarcomas derived from mesenchymal cells (for example, Ewing sarcoma). Unlike hematologic malignancies or brain tumors, solid tumors can arise in any of the three germ cell lineages: mesoderm, endoderm or ectoderm (Figure 1). For example, retinoblastoma and neuroblastoma arise from ectodermally derived cellular lineages, rhabdomyosarcoma and osteosarcoma from mesodermally derived lineages, and hepatoblastoma and pediatric gastrointestinal stromal tumors from endodermally derived lineages. Pediatric solid tumors provide a unique opportunity to study the effect of cellular origin, developmental stage and other intrinsic cellular features that predispose some cells to malignant transformation as a result of distinct combinations of genetic lesions.

The first step in elucidating why some cells are more susceptible to malignant transformation at particular developmental stages with distinct oncogenic lesions than other cells is to define the genomic landscape of each tumor type. A clear understanding of the underlying genetic lesions is essential to parse out the contribution of developmental stage, lineage or other intrinsic cellular features such as the epigenome. Although the genetics of pediatric solid tumors has been studied since the identification of the first human tumor suppressor gene (RB1) in 1986, ${ }^{1}$ we have only recently started to assemble a more comprehensive description of the genomic landscape of these diverse developmental tumors.
Recent advances in pediatric solid tumor genomics have been made possible with the introduction of next-generation sequencing (NGS) technologies and the development of efficient data analysis pipelines to identify complex somatic mutations in pediatric solid tumors. ${ }^{2}$ The genomic landscape of pediatric solid tumors is as complex and diverse as their cellular origins, histopathologic features and clinical presentation. In this review, we provide examples of this diversity and highlight how NGS can provide new insights into the cellular origins of these developmental tumors. We also review associations between genomic and epigenomic features of cancer cells and how those relationships can advance our understanding of the cellular origins of cancer in efforts to identify novel druggable pathways. These findings are incorporated into a unifying model of cellular pliancy that provides a framework for answering fundamental questions in cancer biology. Finally, we present an overview of recent efforts to incorporate cancer genomics into clinical practice for pediatric solid tumors.

\section{DIVERSITY OF PEDIATRIC SOLID TUMORS}

\section{Cellular diversity}

Pediatric solid tumors are remarkably diverse in their histologic features. For example, retinoblastoma tumor cells organize into spheres and extend neuronal processes into the center of the sphere. These processes contain synaptic vesicles, form synaptic junctions and accumulate neurotransmitters. ${ }^{3}$ In contrast, rhabdomyosarcoma tumor cells contain abundant glycogen and have myofibrils reminiscent of skeletal muscle. ${ }^{4}$ Cellular features are often used to infer the cell of origin for pediatric solid tumors. However, evidence of neuronal differentiation in retinoblastoma or muscle differentiation in rhabdomyosarcoma does not prove their cellular origins. Oncogenes and tumor suppressor genes that initiate and promote tumorigenesis also have important roles in

\footnotetext{
${ }^{1}$ Department of Computational Biology and Bioinformatics, St. Jude Children's Research Hospital, Memphis, TN, USA; ${ }^{2}$ Department of Oncology, St. Jude Children's Research Hospital, Memphis, TN, USA; ${ }^{3}$ Department of Developmental Neurobiology, St. Jude Children's Research Hospital, Memphis, TN, USA and ${ }^{4}$ Howard Hughes Medical Institute, Chevy Chase, MD, USA. Correspondence: Dr MA Dyer, Department of Developmental Neurobiology, MS 323, St. Jude Children's Research Hospital, 262 Danny Thomas Place, Memphis 38105-3678, TN, USA.

E-mail: michael.dyer@stjude.org

Received 9 October 2014; revised 9 December 2014; accepted 9 December 2014; published online 2 February 2015
} 


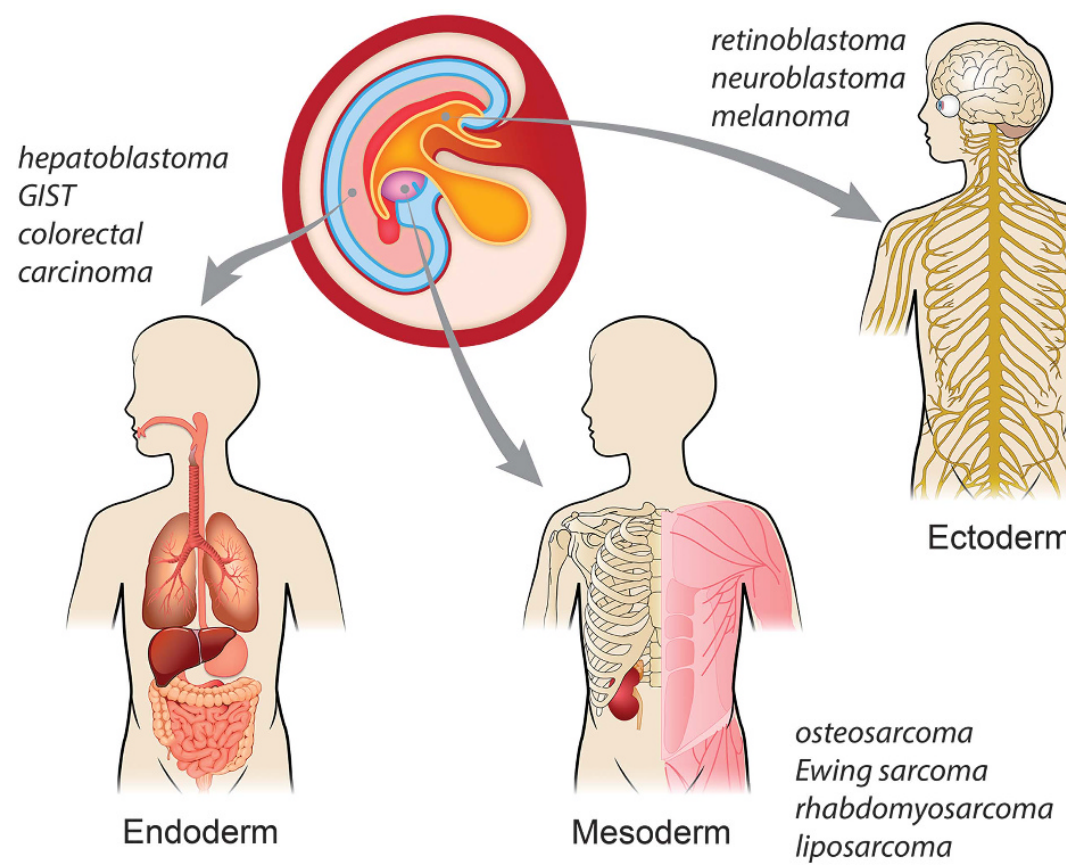

Figure 1. Pediatric solid tumors have diverse developmental origins. Unlike hematologic malignancies and brain tumors, pediatric solid tumors can arise from any of the three germ layers in the embryo. Representative examples are highlighted for each lineage.

normal developmental processes. Thus, as individual cells undergo malignant transformation, they may simultaneously undergo changes in cell fate specification and differentiation. For example, $R B 1$ inactivation contributes to retinoblastoma initiation by promoting cell cycle progression, but the RB1 protein is also required for rod photoreceptor differentiation. ${ }^{5}$ Notably, these roles are cell-type and developmental-stage specific, and there are similar examples for almost every well-characterized oncogene and tumor suppressor gene.

Another important consideration in cellular diversity and malignant transformation is developmental competence. ${ }^{6}$ Pediatric solid tumors arise in complex and dynamic developing tissues as multipotent progenitor cells undergoing unidirectional changes in their intrinsic competence to produce differentiated cell types. ${ }^{6}$ These are some of the most dynamic and robust cellular events in biology, and they pose unique challenges while identifying the cellular origin of pediatric solid tumors. For example, different cellular populations of mesoderm-derived progenitor cells may be heterogeneous in their developmental bias toward muscle, adipose or osteogenic cell fates. When a tumor-initiating mutation occurs in those distinct progenitor cell populations, the consequence may vary dramatically depending on the competence of that cell at that particular stage of development. For example, in one cell population, an oncogenic mutation may result in a tumor with cellular features of skeletal muscle such as rhabdomyosarcoma, whereas in another, the same mutation may lead to rapid cell death or a tumor with osteogenic features (Figure 2). Indeed, recent studies in genetically engineered mouse models show that perturbations in the hedgehog pathway in the adipose lineage can lead to rhabdomyosarcoma with features of skeletal muscle typical of human rhabdomyosarcoma. ${ }^{7}$ Thus, the competence of individual progenitor cells and the specific oncogenic mutations affect when and where pediatric solid tumors arise during development.

The interplay between the specific tumor mutations and differentiation programs, combined with the dynamic intrinsic cellular competence, make it difficult to pinpoint a cell of origin from the molecular or cellular features of the resulting tumors. Instead, it is more informative to study cell fate specification and differentiation in cellular lineages that potentially contribute to pediatric solid tumors and the effect of specific genetic lesions in those lineages at different stages of development. The first step is to define the genetic lesions in different pediatric solid tumors; the inter- and intra-tumor heterogeneity; and the order of events that cause malignant transformation during the development of the retina, bone, neural crest, muscle and other cellular lineages.

\section{Clinical diversity}

In addition to the cellular diversity, clinical diversity can also provide important clues about the developmental origins of pediatric solid tumors. First, the age at diagnosis is often distinct for particular tumor types. Retinoblastomas are among the most common tumors in infants and are rarely, if ever, diagnosed after 5 years of age. Retinal progenitor cell proliferation occurs during the first two trimesters, and there are reports of premature infants diagnosed with retinoblastoma. ${ }^{8}$ These observations, combined with studies on genetically engineered mouse models, suggest that retinoblastomas likely initiate from a retinal progenitor cell in utero and the time from tumor initiation to diagnosis ranges from a few months to a few years. ${ }^{9,10}$ These features associated with the disease presentation of retinoblastoma raise intriguing developmental questions. First, if all tumors initiate in utero during a 3-4month period of retinogenesis, why do some retinoblastomas progress rapidly, leading to diagnosis at birth, whereas others require several years to grow to the same size? In genetically engineered mouse models of retinoblastoma, small clusters of proliferating cells that represent preneoplastic lesions can be identified throughout the retina at the final stages of retinal maturation. ${ }^{11,12}$ However, only a small subset of those preneoplastic lesions progresses to form retinoblastoma. It was previously thought that this was due to the requirement of secondary and tertiary genetic lesions after inactivation of the $R b 1$ gene. However, genomic analyses of human and mouse retinoblastomas have shown that this is not the case. ${ }^{13,14}$ It is possible that only a subset of the preneoplastic lesions progress to retinoblastoma because of specific requirements involving the microenvironment, such as juxtaposition to retinal vasculature. Alternatively, the 
a
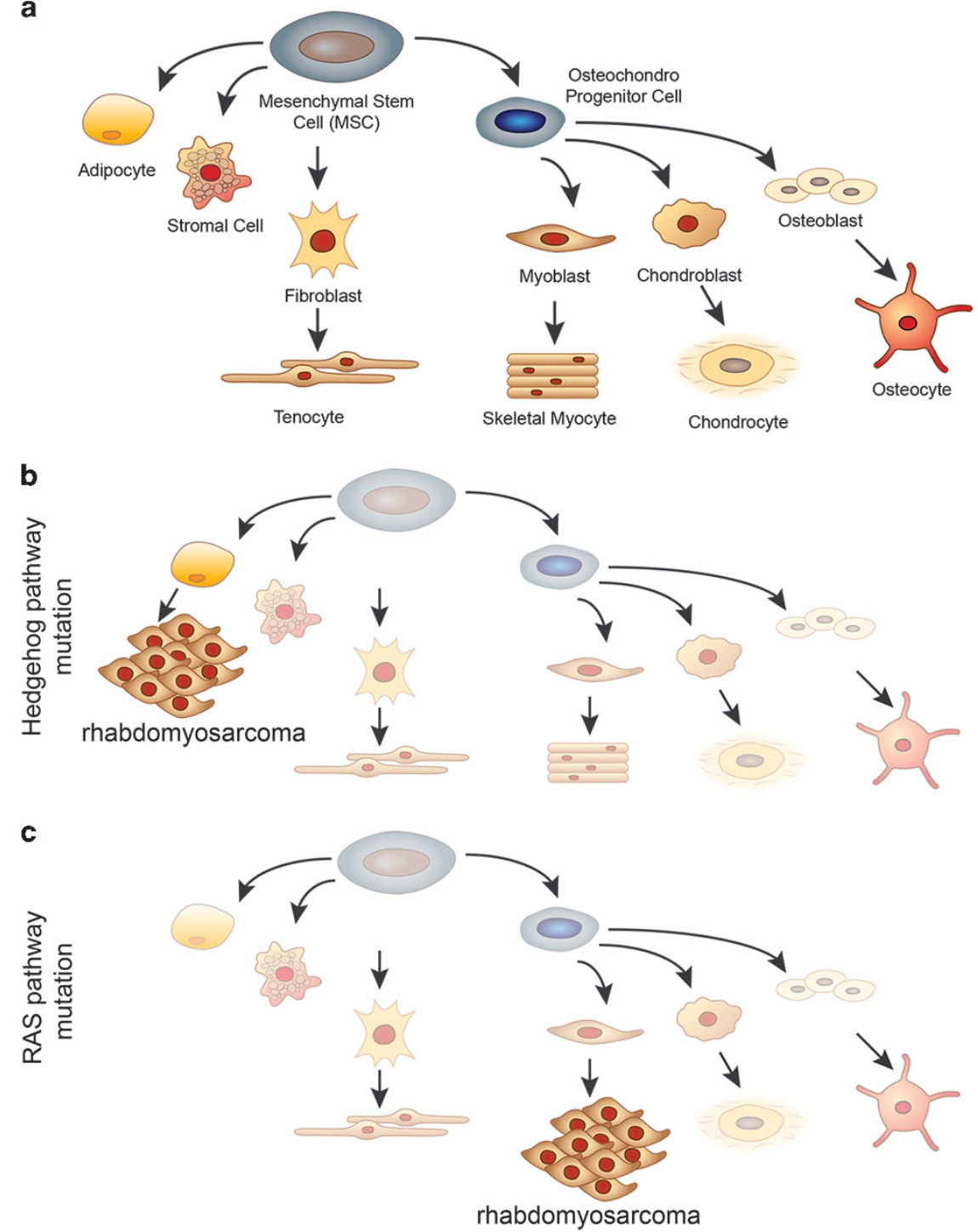

Figure 2. Pediatric solid tumors have diverse cellular origins with unique genetic lesions. (a) A simplified lineage diagram for several mesodermally derived lineages including those relevant to pediatric solid tumors such as bone, muscle and adipose. It is not known which cell lineages or stages are the origins of pediatric solid tumors and there are several alternative models. (b) If the genetic lesions occur at equal frequency across cellular lineages and developmental stages, then one model predicts that particular cells at distinct stages of development are more susceptible to malignant transformation than others. In this example, hedgehog pathway mutations only lead to malignant transformation in the adipose lineage and those cells adopt differentiated features of muscle. Thus, one cannot necessarily infer the cell of origin based on the differentiation features of the tumor itself. (c) The susceptibility to malignant transformation is pathway-dependent. The same adipose lineage is not susceptible to malignant transformation following oncogenic activation of the RAS pathway in this model. Instead, it is the muscle lineage that gives rise to RAS mutant tumors. Only by studying the developmental origins of pediatric solid tumors can we begin to elucidate the cell and developmental stage-specific susceptibility to individual oncogenic lesions.

intrinsic developmental competence or other cell-intrinsic properties such as the epigenome may affect the probability of a particular clone of preneoplastic retinal cells progressing to retinoblastoma. This example highlights the importance of genomic analyses in retinoblastoma. Before the advent of whole-genome sequencing analysis for retinoblastoma, the conventional wisdom was that some retinoblastomas take years to progress because they must acquire additional genetic lesions in key cancer pathways. With that model disproved, researchers are now exploring alternative hypotheses related to developmental microenvironment and epigenetics.

In contrast to retinoblastoma, osteosarcoma is most commonly diagnosed in adolescents and young adults. The peak incidence of osteosarcoma coincides with rapid growth of the skeletal system during puberty. ${ }^{15}$ Females under 15 years of age have a higher incidence of osteosarcoma and the incidence of the disease has an earlier peak in females when compared with males (12 versus 16 years), correlating with an earlier age of onset of puberty. ${ }^{15}$ Osteoblasts likely undergo rapid expansion during this period of development, making them more susceptible to malignant transformation and osteosarcoma formation. However, it has not yet been proven that osteoblasts are the cell of origin for osteosarcoma. It is possible that a completely different lineage adopts an osteogenic phenotype as a result of somatic mutations. Further, changes in hormone levels, cytokines or chemokines in adolescents may contribute to the initiation and progression of osteosarcoma. ${ }^{16}$

As with retinoblastoma, the genomic analysis of osteosarcoma has provided an important foundation for exploring cellular origins and intrinsic and extrinsic factors that may contribute to 
bone tumor formation. Whole-genome sequencing studies show that the p53 pathway is inactivated in virtually all osteosarcomas. ${ }^{17}$ Although TP53 inactivation is not unusual, the mechanism of inactivation is unique. The majority of TP53 mutations in osteosarcomas are translocations in the first intron of the gene. ${ }^{17}$ This mechanism is so prevalent that some osteosarcomas have two independent structural variations in TP53. ${ }^{17}$ To the best of our knowledge, this type of TP53 mutation occurs only in a rare subset of prostate cancers. ${ }^{18,19}$ For osteosarcoma, whole-genome sequencing was required to elucidate this important mechanism of TP53 inactivation. Careful analysis of mutant allele frequency and tumor heterogeneity showed that TP53 mutations are initiating genetic events in osteosarcoma. ${ }^{17}$ These findings highlight a paradox. In many tumor types, inactivation of the p53 pathway can lead to an increase in translocations and other chromosomal events because of perturbations in DNA damage checkpoint control. However, many pediatric osteosarcomas are initiated through a structural variation occurring at very high frequency in TP53 itself. Why are TP53-inactivating translocations such a common mechanism in osteosarcoma and how do they occur at such a high rate even before p53 is mutated? One intriguing possibility is that the cell of origin for osteosarcoma has a higher rate of chromosomal lesions and structural variations than do other cellular lineages. During bone development, the normal DNA damage checkpoints might be suppressed to facilitate rapid expansion of bone precursor cells. Thus, the genomic analysis of osteosarcoma has provided a valuable clue about the genome maintenance in the cell of origin for osteosarcoma.

Although findings from genomic studies on osteosarcoma and retinoblastoma are very different and the clinical features of the diseases are also distinct, there is one similarity. For both osteosarcoma and retinoblastoma, it has been possible to formulate new testable hypotheses about their origins by incorporating genomic data into the context of cellular and developmental processes. Thus, genomic data can provide the foundation for new areas of investigation.

\section{Genetic diversity}

Osteosarcoma and retinoblastoma represent two ends of the continuum of the genomic landscape of pediatric solid tumors. Retinoblastomas have 14.5 (median) structural variations (SVs) per tumor (range 0-96), 10\% of their genome is involved in copy number variations (CNVs) (range 0-95\%) and have an average of 3.5 single nucleotide variations in coding regions (range $0-32$ ). Translocations that are predicted to produce in-frame fusion proteins are rare in retinoblastoma. In contrast, osteosarcomas have 266 SVs per tumor (range $47-1135$ ), 50\% of their genome is involved in CNVs (range 3-89\%) and have 46 single nucleotide variations in coding regions (range 16-237). On average, 8.5 translocations per tumor (median value) produce a novel fusion protein in osteosarcoma.

One explanation for the differences in genomic data between both solid tumor types is that retinoblastomas have stable genomes and osteosarcomas have unstable genomes. However, one needs to be cautious while using genomic data to draw conclusions about genome stability. Usually, the genomic landscape of a tumor is analyzed at a single timepoint and aneuploidy and abundant SVs and CNVs in tumors are thought to arise because of genomic instability; that is, tumors cells can accumulate sequential genetic lesions at a higher rate than do normal cells. Although this may be true in most cases, other acute events such as chromothripsis also need to be considered. ${ }^{13,20}$ Chromothripsis is thought to be a shattering of individual chromosomes, which are then reassembled in a disorganized manner. Importantly, chromothripsis is an acute event rather than a progressive accumulation of chromosomal lesions and is distinct from chromosomal instability. Indeed, the genome may be stable before and after such acute events. It is important to distinguish between the gradual accumulation of genetic lesions with each round of tumor cell division and more acute events such as chromothripsis because of the implications for cellular origins and the intrinsic and extrinsic properties of tumors. For example, genome instability may result from elevated DNA damage due to increased oxidative stress and/or defects in DNA damage repair. In contrast, chromothripsis may arise because of genomic crisis occurring at a particular moment in tumor progression and cells may show no significant defect in oxidative stress or DNA repair. Sequential genomic analyses of individual patient tumors or orthotopic tumor xenografts will allow investigators to distinguish between these and other mechanisms that contribute to alterations in the genomic landscape in cancer. ${ }^{14}$

A long-held belief in adult solid tumor genetics is that tumors with high rates of SVs and CNVs are more aggressive than those with more stable genomes. However, it is not clear whether the high rate of somatic chromosomal lesions and aneuploidy is due to the genomic instability itself or the result of perturbations in underlying pathways that also make the tumors more aggressive. To date, no correlation has been reported between somatic mutations (single nucleotide variations, SVs and CNVs) and outcomes of children with neuroblastoma, osteosarcoma, rhabdomyosarcoma, Ewing sarcoma, adrenocortical carcinoma, retinoblastoma or melanoma. $4,13,14,17,21$ For example, patients with alveolar rhabdomyosarcomas (ARMS) have fewer single nucleotide variations, SVs and CNVs than those with embryonal rhabdomyosarcomas, but the outcome of patients with ARMS is generally worse than that for patients with embryonal rhabdomyosarcoma. ${ }^{22-25}$ Among pediatric bone tumors, genomic lesions are far more common in patients with osteosarcomas than in those with Ewing sarcoma, but they both have comparable overall survival rates. ${ }^{17,26}$ As more tumors are analyzed with NGS, it will be important to elucidate the relation among particular genetic lesions, cellular properties, developmental lineage, intrinsic and extrinsic factors, and genomic stability. This important area of future research is being developed as a direct result of the pediatric solid tumor sequencing efforts over the last 5 years.

Another interesting area of research related to the overall number of somatic mutations in pediatric solid tumors is translocation-driven tumors. Ewing sarcomas are initiated by EWS-FLI1 translocations, and WGS of 112 tumors and matched germline tissue revealed that the tumors have very few secondary genetic lesions. ${ }^{27}$ Similarly, ARMS are initiated by PAX3/7-FOXO translocations and have few secondary genetic lesions. ${ }^{4}$ These data suggest that fusion proteins are the major oncogenic drivers in those tumors, high rates of somatic SVs and CNVs are not required for tumorigenesis, and the cellular context is likely a key factor in malignant transformation of Ewing sarcoma and ARMS.

In addition to these striking differences in the genomic landscape of oncogenic fusion protein-driven pediatric solid tumors, several other unique patterns of DNA mutations contribute to our understanding of cellular origins and the unique developmental context that predisposes some cellular lineages to malignant transformation. Two striking examples of such patterns in pediatric solid tumors are chromothripsis and kataegis. ${ }^{20,28-30}$ These recently discovered patterns of mutation that were originally revealed from NGS of adult cancers have now been identified in pediatric solid malignancies. ${ }^{13,17,31}$ Chromothripsis may occur when individual chromosomes are sequestered into micronuclei as a result of defects in chromosome segregation. Micronuclear DNA replication can become uncoupled from chromosome replication in the nucleus. Thus, if nuclear DNA replication is complete before micronuclear DNA replication and the cell proceeds through mitosis, the replicating chromosome in the micronucleus may become fragmented during chromosomal segregation in mitosis. ${ }^{28}$ The reassembly of fragmented 
chromosome results in a pattern of SVs and CNVs, which are the hallmarks of chromothripsis. In some pediatric solid tumors, chromothripsis can initiate tumorigenesis. For example, retinoblastomas can initiate with chromothripsis on chromosome 13, which results in inactivation of the $R B 1$ gene. ${ }^{13}$ Moreover, some tumor types have higher rates of chromothripsis than other tumor types. Chromothripsis was found in 3\% of retinoblastoma, $4.5 \%$ of Ewing sarcoma, $10.5 \%$ in adrenocortical carcinomas, $20 \%$ of osteosarcomas, $18 \%$ of stage 3 and 4 neuroblastomas and none in rhabdomysarcoma. $413,14,17,20,27,31$ There was no significant association between TP53 status and chromothripsis in these tumors. It is not known whether this reflects a propensity for missegregation of chromosomes during mitosis and formation of micronuclei, perturbations in the initiation and progression of DNA replication across the genome, defects in checkpoint control, or a combination of these events. A deeper understanding of the underlying molecular mechanisms of chromothripsis and the regulation of those processes across cellular lineages in development may provide important insights into the cellular and developmental origins of pediatric solid tumors.

Even less is known about the underlying molecular mechanisms of kataegis, which involves focal nucleotide hypermutation adjacent to SVs. ${ }^{30}$ As with chromothripsis, kataegis was first identified in adult cancers through NGS and it is associated with some forms of pediatric solid tumors. ${ }^{17}$ Kataegis is relatively common in osteosarcoma (50\%) and adrenocortical carcinomas (31\%) but not in other pediatric solid tumors. All tumors with the kataegis pattern carried mutated TP53. Kataegis is not seen in pediatric solid tumors with low rates of SVs, such as Ewing sarcoma, retinoblastoma and ARMS. However, kataegis is not necessarily found in pediatric solid tumors with high rates of SVs. For example, kataegis has been reported in $50 \%$ of osteosarcomas but not in embryonal rhabdomyosarcomas. ${ }^{4,17}$ Both tumor types have high rates of SVs and CNVs, but differ markedly in the occurrence of chromothripsis and kataegis. Little is known about the association of perturbations in particular DNA repair pathways or homologous recombination pathways and kataegis or their role in normal development of endodermally, mesodermally and ectodermally derived lineages. Unlike chromothripsis, there is no evidence supporting that mutations associated with kataegis contribute to tumor initiation or progression in pediatric solid tumors. That is, kataegis-associated mutations have not been identified in recurrent regions of the genome or in known oncogenic drivers in childhood cancer. However, whole-genome sequencing and detailed analysis of kataegis have been performed in only a small number of pediatric solid tumor genomes, and future studies may reveal a role for kataegis in tumor initiation and progression.

\section{Reprogramming the tumor genome}

The examples described above highlight how genomics can be integrated with histopathology and clinical features of pediatric solid tumors in the context of the underlying developmental biology. Such studies can help advance the field and ask fundamental questions that may have broad implications for cancer biology. In the case of retinoblastoma, if there are barriers to tumor progression and they are not genetic, what are they? Recent advances in stem cell biology may provide a useful framework for exploring processes that lead to reprogramming of the tumor genome. For example, are there any similarities between the barriers to oncogenic transformation and the barriers to reprogramming of somatic cells into induced pluripotent stem cells? It is well established that the major barrier to producing stem cells from differentiated cells is reorganization of the epigenetic landscape. ${ }^{32-35}$ For a given cell type, it is believed that all cells have an equal, albeit low, probability of overcoming those epigenetic barriers. Does this hold true for cancer? Are there similar epigenetic barriers to malignant transformation? It is possible that a single oncogenic event, such as formation of the EWS-FLI1 oncogene, promotes the formation of Ewing sarcoma in a stochastic manner through active epigenetic reprogramming, as seen in the formation of induced pluripotent stem cells from somatic cells (Figure 2). Alternatively, the genetic lesions that initiate a tumor might not reprogram the epigenome, but instead the mutation must occur in a cell with a permissible epigenomic landscape (Figure 2). This latter model can begin to explain the cell-type specificity of particular oncogenic mutations in particular cellular lineages. For example, the EWS-FLI translocation may occur in various cell types at different developmental stages, but only one of those cell types has the appropriate epigenetic landscape that leads to malignant transformation in the presence of the EWS-FLI oncoprotein. Indeed, it has been shown that ectopic expression of the EWS-FLI oncogene is not tolerated in differentiated cells but is tolerated in mesenchymal stem cells where it induces a gene expression profile similar to that found in Ewing sarcoma. ${ }^{36}$ The cellular context is critical in both models. Just as the barriers to reprogramming are dependent on the somatic cell of origin, the barriers to malignant transformation are also a result of specific oncogenic mutations. None of these questions could have been posed without a detailed understanding of the genomic landscape of pediatric solid tumors that has emerged with NGS over the past 5 years.

\section{EPIGENETICS}

One of the most important advances resulting from the genomic analysis of pediatric cancer over the past 5 years was the discovery that epigenetics plays a major role in tumor initiation and progression. DNA methylation patterns and other epigenetic changes have been studied in cancer for decades, but it can be difficult to differentiate epigenetic and genetic contributions without a detailed analysis of individual cancer genomes. In tumors that progress rapidly after the initiating genetic lesion, such as retinoblastoma, genomic analyses show that some tumors progress quickly even without secondary genetic lesions. ${ }^{14}$ An integrated analysis of DNA methylation, gene expression and histone modification in retinoblastoma showed that the expression of several oncogenes and tumor suppressor genes was altered through epigenetic mechanisms. ${ }^{14}$ For example, the SYK oncogene was upregulated in all retinoblastomas and was required for tumorigenesis. ${ }^{14}$ As the epigenomic profiling of normal cell populations during development advances and those reference epigenomes are compared with epigenomes from pediatric solid tumors, we hope to gain an even deeper understanding of the relation among genomic lesions, the epigenome and developmental competence in malignant transformation.

Another unexpected discovery that highlighted the importance of epigenetics in solid tumor progression was that of recurrent mutations in the epigenetic modulators themselves. For example, in retinoblastoma, the only recurrently mutated gene other than $R B 1$ is the epigenetic regulator $B C O R .{ }^{14}$ BCOR interacts with BCL-6 to repress gene expression, and germline mutations in $B C O R$ are associated with the oculofaciocardiodental syndrome. ${ }^{37}$ During development, BCOR is thought to maintain tissue homeostasis and gene silencing through epigenetic mechanisms. ${ }^{38,39}$ In the absence of BCOR, H3K4 and H3K36 methylation increases in mesenchymal stem cells. ${ }^{39}$ It is not known how BCOR contributes to retinal development or tumor progression, but it has now emerged as an important cancer gene that is mutated in 21 different human cancers with frequencies randing from 0.5 to $40 \%$. Further analyses of the epigenome of retinoblastomas with wild-type and mutant $B C O R$ are required to identify the key target genes for tumor progression. 
ATRX is the most commonly mutated epigenetic regulator in childhood cancer. ATRX mutations were previously unknown in pediatric cancer, but after completion of the Pediatric Cancer Genome Project, ${ }^{2}$ ATRX has been identified as one of the most commonly mutated genes in pediatric solid tumors after TP53. Somatic mutations in ATRX occur in $22 \%$ of neuroblastomas, ${ }^{21,40}$ $18 \%$ of adrenocortical carcinomas and $29 \%$ of osteosarcomas. ${ }^{17}$ Although ATRX is an X-linked gene, there is no significant gender bias of mutation status in these cancers. In neuroblastomas, ATRX mutations are associated with age at diagnosis, which is highly predictive of overall survival. ${ }^{21,32}$ In adrenocortical carcinomas, ATRX mutations, which occurred in tumors harboring TP53 mutations, are associated with genome instability, advanced tumor stage and poor outcomes. Interestingly, ATRX mutations have never been found in combination with MYCN amplification in neuroblastoma. Further comprehensive studies are required to determine whether ATRX mutations are an independent prognostic risk factor for neuroblastoma.

In most tumors with ATRX mutations, cells have longer telomeres as a result of alternative lengthening of telomeres, which is a mechanism of telomere maintenance in cancer. ${ }^{41}$ This finding is consistent with the role of ATRX in histone H3.3 deposition at telomeres. Like BCOR, ATRX is also an epigenetic modulator and an X-linked gene. Germline ATRX mutations lead to the X-linked alpha thalassemia/mental retardation syndrome, which is associated with diverse developmental defects. ${ }^{42-49}$ Although alternative lengthening of telomeres and telomere maintenance are important for tumorigenesis, inactivation of ATRX may also contribute to perturbations in other cellular pathways through epigenetic mechanisms. The crystal structure of the ADD domain of ATRX reveals two binding pockets for the histone H3 $\mathrm{N}$-terminal tail. One pocket binds unmodified lysine 4 and the other binds di- or trimethylated lysine $9 .{ }^{50} \mathrm{~A}$ more extensive analysis of the localization of ATRX in the cancer genome and determination of whether the truncated ATRX proteins abundant in neuroblastoma and osteosarcoma have any function will significantly advance our understanding of pediatric solid tumors.

Epigenetics has emerged as a major focus area in pediatric solid tumor research because of the striking changes in the epigenetic landscape after inactivation of tumor suppressor genes such as $R B 1$ and the identification of recurrent mutations in epigenetic regulators such as $B C O R$ and $A T R X$. By integrating these tumor data with epigenomic profiling of normal cells during development, we can develop a new framework to understand why some oncogenic mutations cause specific types of tumors in restricted cell lineages during development.

\section{CELLULAR PLIANCY}

To unify the findings in pediatric solid tumor genomics and integrate them with our understanding of developmental competence and lineage specification, we have developed a new concept called cellular pliancy. We suggest that cellular pliancy is a unique feature of each cell type that determines whether it will be susceptible to malignant transformation after sustaining a particular genetic lesion. The pliancy model provides a framework for understanding why some cell types are more susceptible to malignant transformation than other cell types with particular genetic lesions. The concept of cellular pliancy can also be extended to cell-type-specific degeneration associated with aging or other changes in the microenvironment and cellular homeostasis.

To provide a framework for the pliancy model of transformation and degeneration, we outline five key principles that serve as the foundation for the model.

First, tumor suppressor genes and proto-oncogenes have cell-type-specific and stage-specific roles in development Therefore, the effect of tumor suppressor gene inactivation or proto-oncogene activation on tumor initiation and progression is also likely to be dependent on cellular lineage and developmental stage.

Second, a hallmark of tissue development is that it is mediated by dynamic cellular processes with temporal and spatial heterogeneity. These discrete cellular states are critical determinants of the cellular origins of childhood solid tumors. Specifically, it is possible that individual oncogenic lesions in a particular cellular lineage at a unique developmental stage lead to cancer but other combinations of mutation, lineage and stage are not tolerated.

Third, a major component of this rapidly changing intrinsic cellular milieu during development involves the reorganization of the epigenome of cells. Once a cell commits to a particular fate and activates the transcriptional networks that define that fate, the rest of the genome that is not directly required for differentiation is epigenetically partitioned into silent inaccessible domains and permissive domains that can be reactivated under stress. The partitioning of the genome into inaccessible and permissive domains is cell-type-specific and is a key determinant of the cell-type-specific response to oncogenic mutations, cell stress and other perturbations in normal cell homeostasis.

Fourth, the epigenetic landscape can change in response to oncogenic lesions and, in particular, the partitioning of genomic regions into inaccessible and permissive domains may be altered by particular somatic mutations associated with cancer. Also, the partitioning of the genome may degrade with age and this in turn could affect cancer susceptibility. Somatic mutations in epigenetic regulators may directly affect the partitioning of the epigenome and the maintenance of those critical silent and permissive domains in each cellular lineage.

Fifth, the epigenetic landscape may serve as a fingerprint of the cellular origins of cancer. Although all partitioned domains are not likely to be maintained in the tumor cells because of changes associated with age and/or genetic mutations in cancer cells, a sufficient number may remain to identify the developmental- and lineage-specific remnants of the cell of origin. This could provide an opportunity to identify the cellular origin for pediatric solid tumors and to advance our understanding of the changing epigenome during development.

By combining these principles, we propose that cellular pliancy is the intrinsic susceptibility of a cell to undergo malignant transformation or degeneration. In its simplest form, cellular pliancy is the epigenetic organization of the genome as cells commit to a particular cell fate and activate their differentiation program. Cellular pliancy involves the partitioning of genes that are not required for cellular differentiation or normal cellular homeostasis into silent or permissive chromatin states. We suggest that the partitioning of those genes is cell-type-specific and evolutionarily selected for each cell type. Cells with high pliancy may have more of their genome in an open or permissive chromatin state, thus making them more adaptable in the face of injury, oncogenic lesions or changes in the microenvironment. However, such cells may be more susceptible to malignant transformation, because oncogenes and tumor suppressor genes that must be deregulated for tumor progression can be readily altered after the oncogenic lesion. Cell types with low pliancy are more difficult to transform, but these cells may be more prone to degeneration because they cannot adapt efficiently to changes in homeostasis or environment. For example, rod photoreceptors in the retina are highly susceptible to degeneration but virtually impossible to transform. In contrast, horizontal cells are much more resistant to degeneration but can form retinoblastoma when the $\mathrm{Rb}$ pathway is inactivated. ${ }^{51}$ Indeed, the concept of global partitioning of genes into silent and permissive states may be less important than the individual genes themselves. Thus, the combination of the previously unexplored distribution of genes that are not normally expressed into different chromatin states 
will have predictive power on a cell's susceptibility to undergo malignant transformation or degeneration.

\section{CELLULAR PLIANCY-A 'NUCLEAR SUITCASE'}

We envision that cellular pliancy is analogous to packing a suitcase for a long trip. A seasoned traveler will prepare a suitcase with everything needed for the trip. On a different voyage, the traveler will likely pack entirely different items. As individual cells prepare for their journey down a particular cellular lineage, they must also pack their genome for that particular differentiation program. Evolution and selection have identified the genes that may be needed in specific cells over their lifetime to handle potential changes in the microenvironment or other types of injury or stress. Cells pack their nucleus for their journey by partitioning genes into accessible or silent chromatin domains. Accessible genes are those that may be required in the lifetime of the cell, and silenced genes are those that are essentially left behind. Once a traveler packs a suitcase, he or she must make do with the items on hand. Similarly, once the nucleus is packed, the cell has a limited repertoire of genes and pathways that can be activated as a result of changes in homeostasis, acute injury or stress. A few poor choices could mean the difference between a successful voyage and a fatal one.

According to our 'nuclear suitcase' analogy, cellular pliancy is the choice a cell makes in how it packs its genome to prepare for events that may arise during its lifetime. Those genome-packing decisions have not been extensively studied, because the genes are expressed only under particular stress conditions and most of the focus in the last two decades has been on cell-type-specific gene expression patterns rather than cell-type-specific packing of the genome into permissive and silent domains. The advantage of this model is that it can be directly tested with current technology. One prediction is that oncogenic pathways that are required for malignant transformation will be epigenetically poised in the cell that gives rise to that tumor. Alternatively, the particular genetic lesion results in reprogramming of the epigenetic state of that locus. This theory can be tested for cell-type-specific degeneration. If this is true, it may allow us to define combinations of genetic and epigenetic landscapes that are oncogenic and combinations of stress (for example, oxidative stress) and epigenetic landscapes that predispose cells to degeneration. Armed with this knowledge, we can more effectively identify weaknesses that can be exploited in tumor cells by using molecular targeted therapeutics.

\section{CLINICAL GENOMICS}

The overall survival rate of children with a variety of solid tumors including sarcomas is worse than that for children with other childhood cancers, and there have not been significant improvements in outcome for the vast majority of these children over the past two decades. ${ }^{52}$ For children with recurrent or metastatic neuroblastoma, osteosarcoma, Ewing sarcoma and rhabdomyosarcoma, the overall survival rate is below 30\%. ${ }^{53-56}$ With the widespread use of NGS technology to diagnose pediatric cancers, several efforts are underway to identify somatic and germline mutations in a clinical setting. However, this is an emerging area of investigation and it is not yet clear how the genomic analysis of pediatric solid tumors can be used to individualize cancer therapy. For example, pediatric cancer is rare, and it may be difficult to establish the statistical significance of new therapies for rare subsets of patients receiving individualized treatment based on the mutations found in their tumors. Therefore, if a new therapeutic approach is thought to be efficacious for a genetically defined subgroup of patients, it should be tested in a prospective clinical trial. Unfortunately, with our current understanding of pediatric solid tumor genomes and available molecular targeted therapies, only $12 \%$ of patients (46/380) with pediatric solid tumor patients likely have 'druggable' mutations. ${ }^{57}$ Even this estimate may be overly optimistic, because the presence of a druggable mutation does not necessarily guarantee that the tumor will respond better to a therapy targeted to that lesion than it will to other drug combinations. Also, the definition of a druggable mutation varies significantly across clinical genomic/personalized oncology trials. For example, activating mutations in the ALK gene are thought to be druggable with molecular targeted therapeutics such as crizotinib. ${ }^{58}$ A pediatric phase I trial was completed for crizotinib ${ }^{59}$ and a Phase I/II study is ongoing for children with relapse or refractory ALK mutant tumors (NCT00939770). The inclusion criteria for the Phase I/II study includes patients whose tumors have $A L K$ gene fusions, mutations and amplifications ( $>$ fourfold). In neuroblastomas with $A L K$ mutations, it is not known whether some mutations confer greater sensitivity to crizotinib than others. Moreover, there is relatively little known about the relationship between $A L K$ gene fusions or CNVs and crizotinib sensitivity. There are several different ALK inhibitors in clinical development and the same questions need to be answered for each of those drugs to determine whether ALK is a druggable target in neuroblastoma.

The presence of a druggable mutation in a tumor and the availability of a drug that targets the deregulated pathway does not ensure efficacy in patients. For example, RAS is mutated in a subset of intermediate- and high-risk rhabdomyosarcomas, but none of the nearly 100 drugs that target this pathway show any differential activity on tumors from patients with RAS mutant rhabdomyosarcomas versus those with wild-type $R A S^{4}{ }^{4}$ These examples highlight the importance of integrating basic, translational and clinical research to begin to identify the best drug combinations for particular subgroups of patients.

For the vast majority of pediatric patients with solid tumors, there are no somatic mutations in the tumor that can be exploited with the current arsenal of molecular targeted therapeutics. However, clinical genomics will provide a more complete catalog of germline and somatic mutations in pediatric solid tumors and contribute important insights into tumor heterogeneity and the clonal evolution of cancer. It will be important to incorporate whole-genome sequencing, whole-exome sequencing and RNA sequencing in future clinical genomics efforts to identify potentially druggable mutations and to advance our understanding of the genetic underpinnings of pediatric solid tumors. Complex somatic genomic lesions such as chromothripsis, kataegis and the structural variations in TP53 found in osteosarcoma would be missed by targeted resequencing approaches such as those used in the iCAT study (NCT01853345), wholeexome sequencing alone ${ }^{60}$ or exome sequencing combined with RNA sequencing (NCT02162732).

\section{CONFLICT OF INTEREST}

The authors declare no conflict of interest.

\section{REFERENCES}

1 Friend SH, Bernards R, Rogelj S, Weinberg RA, Rapaport JM, Albert DM et al. A human DNA segment with properties of the gene that predisposes to retinoblastoma and osteosarcoma. Nature 1986; 323: 643-646.

2 Downing JR, Wilson RK, Zhang J, Mardis ER, Pui CH, Ding L et al. The Pediatric Cancer Genome Project. Nat Genet 2012; 44: 619-622.

3 Johnson DA, Zhang J, Frase S, Wilson M, Rodriguez-Galindo C, Dyer MA. Neuronal differentiation and synaptogenesis in retinoblastoma. Cancer Res 2007; 67: 2701-2711.

4 Chen X, Stewart E, Shelat AA, Qu C, Bahrami A, Hatley M et al. Targeting oxidative stress in embryonal rhabdomyosarcoma. Cancer Cell 2013; 24: 710-724. 
5 Zhang J, Gray J, Wu L, Leone G, Rowan S, Cepko CL et al. Rb regulates proliferation and rod photoreceptor development in the mouse retina. Nat Genet 2004; 36 351-360.

6 Cepko CL, Austin CP, Yang X, Alexiades M, Ezzeddine D. Cell fate determination in the vertebrate retina. Proc Natl Acad Sci USA 1996; 93: 589-595.

7 Hatley ME, Tang W, Garcia MR, Finkelstein D, Millay DP, Liu N et al. A mouse model of rhabdomyosarcoma originating from the adipocyte lineage. Cancer Cell 2012; 22: $536-546$.

8 Abramson DH, Schefler AC, Beaverson KL, Rollins IS, Ruddat MS, Kelly CJ. Rapid growth of retinoblastoma in a premature twin. Arch Ophthalmol 2002; 120 1232-1233.

9 Macpherson D, Dyer MA. Retinoblastoma: from the two-hit hypothesis to targeted chemotherapy. Cancer Res 2007; 67: 7547-7550.

10 Dyer MA, Bremner R. The search for the retinoblastoma cell of origin. Nat Rev Cancer 2005; 5: 91-101.

11 McEvoy J, Flores-Otero J, Zhang J, Nemeth K, Brennan R, Bradley C et al. Coexpression of normally incompatible developmental pathways in retinoblastoma genesis. Cancer Cell 2011; 20: 260-275.

12 Donovan SL, Schweers B, Martins R, Johnson D, Dyer MA. Compensation by tumor suppressor genes during retinal development in mice and humans. BMC Biol 2006; 4: 14.

13 McEvoy J, Nagahawatte P, Finkelstein D, Richards-Yutz J, Valentine M, Ma J et al. RB1 gene inactivation by chromothripsis in human retinoblastoma. Oncotarget 2014; 5: 438-450.

14 Zhang J, Benavente CA, McEvoy J, Flores-Otero J, Ding L, Chen X et al. A novel retinoblastoma therapy from genomic and epigenetic analyses. Nature 2012; 481: 329-334.

15 Mirabello L, Troisi RJ, Savage SA. Osteosarcoma incidence and survival rates from 1973 to 2004: data from the Surveillance, Epidemiology, and End Results Program. Cancer 2009; 115: 1531-1543.

16 Ruza E, Sotillo E, Sierrasesumaga L, Azcona C, Patino-Garcia A. Analysis of polymorphisms of the vitamin $D$ receptor, estrogen receptor, and collagen lalpha1 genes and their relationship with height in children with bone cancer J Pediatr Hematol Oncol 2003; 25: 780-786.

17 Chen X, Bahrami A, Pappo A, Easton J, Dalton J, Hedlund E et al. Recurrent somatic structural variations contribute to tumorigenesis in pediatric osteosarcoma. Cell Rep 2014; 7: 104-112.

18 Baca SC, Prandi D, Lawrence MS, Mosquera JM, Romanel A, Drier Y et al. Punctuated evolution of prostate cancer genomes. Cell 2013; 153: 666-677.

19 Berger MF, Lawrence MS, Demichelis F, Drier Y, Cibulskis K, Sivachenko AY et al. The genomic complexity of primary human prostate cancer. Nature 2011; 470: 214-220.

20 Stephens PJ, Greenman CD, Fu B, Yang F, Bignell GR, Mudie LJ et al. Massive genomic rearrangement acquired in a single catastrophic event during cancer development. Cell 2011; 144: 27-40.

21 Cheung NK, Zhang J, Lu C, Parker M, Bahrami A, Tickoo SK et al. Association of age at diagnosis and genetic mutations in patients with neuroblastoma. JAMA 2012 307: 1062-1071.

22 Scrable H, Cavenee W, Ghavimi F, Lovell M, Morgan K, Sapienza C. A model for embryonal rhabdomyosarcoma tumorigenesis that involves genome imprinting Proc Natl Acad Sci USA 1989; 86: 7480-7484.

23 Ognjanovic S, Linabery AM, Charbonneau B, Ross JA. Trends in childhood rhabdomyosarcoma incidence and survival in the United States, 1975-2005. Cancer 2009; 115: 4218-4226.

24 Barr FG. Chromosomal translocations involving paired box transcription factors in human cancer. Int J Biochem Cell Biol 1997; 29: 1449-1461.

25 Raney RB, Anderson JR, Barr FG, Donaldson SS, Pappo AS, Qualman SJ et al. Rhabdomyosarcoma and undifferentiated sarcoma in the first two decades of life: a selective review of intergroup rhabdomyosarcoma study group experience and rationale for Intergroup Rhabdomyosarcoma Study V. J Pediatr Hematol Oncol 2001; 23: 215-220.

26 Howlader N, Noone AM, Krapcho M, Garshell J, Neyman N, Altekruse SF et al. SEER Cancer Statistics Review, 1975-2010, NCI. 2013; Available from: http://seer.cancer. gov/csr/1975_2010/.

27 Tirode F, Surdez D, Ma X, Parker M, Le Deley MC, Bahrami A et al. Genomic landscape of Ewing sarcoma defines an aggressive subtype with co-association of STAG2 and TP53 mutations. Cancer Discov 2014; 4: 1342-1353.

28 Crasta K, Ganem NJ, Dagher R, Lantermann AB, Ivanova EV, Pan Y et al. DNA breaks and chromosome pulverization from errors in mitosis. Nature 2012 482: $53-58$.

29 Lada AG, Dhar A, Boissy RJ, Hirano M, Rubel AA, Rogozin IB et al. AID/APOBEC cytosine deaminase induces genome-wide kataegis. Biol Direct 2012; 7: 47.

30 Nik-Zainal S, Alexandrov LB, Wedge DC, Van Loo P, Greenman CD, Raine K et al. Mutational processes molding the genomes of 21 breast cancers. Cell 2012; 149: 979-993.
31 Molenaar JJ, Koster J, Zwijnenburg DA, van Sluis P, Valentijn LJ, van der Ploeg I et al. Sequencing of neuroblastoma identifies chromothripsis and defects in neuritogenesis genes. Nature 2012; 483: 589-593.

32 Hanna JH, Saha K, Jaenisch R. Pluripotency and cellular reprogramming: facts, hypotheses, unresolved issues. Cell 2010; 143: 508-525.

33 Mikkelsen TS, Hanna J, Zhang X, Ku M, Wernig M, Schorderet P et al. Dissecting direct reprogramming through integrative genomic analysis. Nature 2008; 454: 49-55.

34 Singhal N, Graumann J, Wu G, Arauzo-Bravo MJ, Han DW, Greber B et al. Chromatin-remodeling components of the BAF complex facilitate reprogramming. Cell 2010; 141: 943-955.

35 Orkin SH, Hochedlinger K. Chromatin connections to pluripotency and cellular reprogramming. Cell 2011; 145: 835-850.

36 Riggi N, Suva ML, Suva D, Cironi L, Provero P, Tercier S et al. EWS-FLI-1 expression triggers a Ewing's sarcoma initiation program in primary human mesenchyma stem cells. Cancer Res 2008; 68: 2176-2185.

$37 \mathrm{Ng} \mathrm{D}$, Thakker N, Corcoran CM, Donnai D, Perveen R, Schneider A et al. Oculofaciocardiodental and Lenz microphthalmia syndromes result from distinct classes of mutations in BCOR. Nat Genet 2004; 36: 411-416.

38 Wamstad JA, Corcoran CM, Keating AM, Bardwell VJ. Role of the transcriptional corepressor Bcor in embryonic stem cell differentiation and early embryonic development. PLoS One 2008; 3: e2814.

39 Fan Z, Yamaza T, Lee JS, Yu J, Wang S, Fan G et al. BCOR regulates mesenchymal stem cell function by epigenetic mechanisms. Nat Cell Biol 2009; 11: 1002-1009.

40 Cheung NK, Dyer MA. Neuroblastoma: developmental biology, cancer genomics and immunotherapy. Nat Rev Cancer 2013; 13: 397-411.

41 Pickett $\mathrm{H}$, Reddel R. Alternative Lengthening of Telomeres in Human Cancer. In: Hiyama K editor. Telomeres and Telomerase in Cancer. Humana: New York, 2009.

42 Gibbons RJ, Pellagatti A, Garrick D, Wood WG, Malik N, Ayyub H et al. Identification of acquired somatic mutations in the gene encoding chromatinremodeling factor ATRX in the alpha-thalassemia myelodysplasia syndrome (ATMDS). Nat Genet 2003; 34: 446-449.

43 Xue Y, Gibbons R, Yan Z, Yang D, McDowell TL, Sechi S et al. The ATRX syndrome protein forms a chromatin-remodeling complex with Daxx and localizes in promyelocytic leukemia nuclear bodies. Proc Natl Acad Sci USA 2003; 100: 10635-10640.

44 Steensma DP, Higgs DR, Fisher CA, Gibbons RJ. Acquired somatic ATRX mutations in myelodysplastic syndrome associated with alpha thalassemia (ATMDS) convey a more severe hematologic phenotype than germline ATRX mutations. Blood 2004; 103: 2019-2026.

45 Costa DB, Fisher CA, Miller KB, Pihan GA, Steensma DP, Gibbons RJ et al. A novel mutation in the last exon of ATRX in a patient with alpha-thalassemia myelodysplastic syndrome. Eur J Haematol 2006; 76: 53.

46 Garrick D, Sharpe JA, Arkell R, Dobbie L, Smith AJ, Wood WG et al. Loss of Atrx affects trophoblast development and the pattern of $\mathrm{X}$-inactivation in extraembryonic tissues. PLoS Genet 2006; 2: e58.

47 Lewis PW, Elsaesser SJ, Noh KM, Stadler SC, Allis CD. Daxx is an H3.3-specific histone chaperone and cooperates with ATRX in replication-independent chromatin assembly at telomeres. Proc Natl Acad Sci USA 2010; 107: 14075-14080.

48 Gibbons RJ, McDowell TL, Raman S, O'Rourke DM, Garrick D, Ayyub $\mathrm{H}$ et al. Mutations in ATRX, encoding a SWI/SNF-like protein, cause diverse changes in the pattern of DNA methylation. Nat Genet 2000; 24: 368-371.

49 Argentaro A, Yang JC, Chapman L, Kowalczyk MS, Gibbons RJ, Higgs DR et al. Structural consequences of disease-causing mutations in the ATRX-DNMT3DNMT3L (ADD) domain of the chromatin-associated protein ATRX. Proc Natl Acad Sci USA 2007; 104: 11939-11944.

50 Eustermann S, Yang JC, Law MJ, Amos R, Chapman LM, Jelinska C et al. Combinatorial readout of histone $\mathrm{H} 3$ modifications specifies localization of ATRX to heterochromatin. Nat Struct Mol Biol 2011; 18: 777-782.

51 Ajioka I, Martins RA, Bayazitov IT, Donovan S, Johnson DA, Frase $S$ et al. Differentiated horizontal interneurons clonally expand to form metastatic retinoblastoma in mice. Cell 2007; 131: 378-390.

52 Smith MA, Altekruse SF, Adamson PC, Reaman GH, Seibel NL. Declining childhood and adolescent cancer mortality. Cancer 2014; 120: 2497-2506.

53 Pappo AS, Anderson JR, Crist WM, Wharam MD, Breitfeld PP, Hawkins D et al. Survival after relapse in children and adolescents with rhabdomyosarcoma: A report from the Intergroup Rhabdomyosarcoma Study Group. J Clin Oncol 1999; 17: 3487-3493.

54 Leavey PJ, Mascarenhas L, Marina N, Chen Z, Krailo M, Miser J et al. Prognostic factors for patients with Ewing sarcoma (EWS) at first recurrence following multi-modality therapy: A report from the Children's Oncology Group. Pediatr Blood Cancer 2008; 51: 334-338.

55 Kempf-Bielack B, Bielack SS, Jurgens H, Branscheid D, Berdel WE, Exner GU et al. Osteosarcoma relapse after combined modality therapy: an analysis of unselected patients in the Cooperative Osteosarcoma Study Group (COSS). J Clin Oncol 2005 23: $559-568$. 
56 Cole KA, Maris JM. New strategies in refractory and recurrent neuroblastoma: translational opportunities to impact patient outcome. Clin Cancer Res 2012; 18: 2423-2428.

57 Shukla N, Ameur N, Yilmaz I, Nafa K, Lau CY, Marchetti A et al. Oncogene mutation profiling of pediatric solid tumors reveals significant subsets of embryonal rhabdomyosarcoma and neuroblastoma with mutated genes in growth signaling pathways. Clin Cancer Res 2012; 18: 748-757.

58 Carpenter EL, Mosse YP. Targeting ALK in neuroblastoma--preclinical and clinical advancements. Nat Rev Clin Oncol 2012; 9: 391-399.

59 Mosse YP, Lim MS, Voss SD, Wilner K, Ruffner K, Laliberte J et al. Safety and activity of crizotinib for paediatric patients with refractory solid tumours or anaplastic large-cell lymphoma: a Children's Oncology Group phase 1 consortium study. Lancet Oncol 2013; 14: 472-480.
60 Scollon S, Bergstrom K, Kerstein RA, Wang T, Hilsenbeck SG, Ramamurthy U et al. Obtaining informed consent for clinical tumor and germline exome sequencing of newly diagnosed childhood cancer patients. Genome Med 2014; 6: 69.

(C) This work is licensed under a Creative Commons AttributionConCommercial-NoDerivs 4.0 International License. The images or other third party material in this article are included in the article's Creative Commons license, unless indicated otherwise in the credit line; if the material is not included under the Creative Commons license, users will need to obtain permission from the license holder to reproduce the material. To view a copy of this license, visit http:// creativecommons.org/licenses/by-nc-nd/4.0/ 